\title{
ESTRATEGLAS DE COMERCIALIZACIÓN DEL SACHA INCHI
}

\section{.}

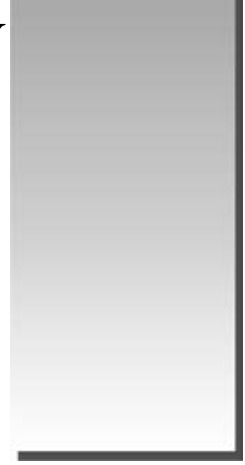

Pedro L. Tito Huamaní(*)

E-mail: pedrotito18@hotmail.com

Elena Bautista Flores(")

E-mail: eibautistaflores@yahoo.com

\begin{abstract}
RESUMEN
La presente investigación tiene como propósito de estudio desarrollar estrategias viables para posicionar y comercializar el Sacha Inchi y sus derivados en el mercado de la gran Lima.

El Sacha Inchi es una planta nativa de la Amazonía peruana, descrita por primera vez como especie en el año 1753 por el naturalista Linneo, de ahí su nombre científico Plukenetia volúbilis linneo.

En el Perú se conoce de su existencia desde el incanato. Así, hasta nuestros días, ha tomado diferentes nombres: Sacha Inchic, palabra quechua que significa maní del monte o silvestre; Amui, término utilizado por las tribus aborígenes de la amazonía; Sacha Inchi, maní del monte, maní del Inca e Inca peanut.

En la actualidad, conocida también como el maní de los Incas, es la oleaginosa natural con mayor contenido de omega, un ácido graso esencial para la vida del ser humano. Está compuesto de $48.60 \%$ de ácido graso esencial alfa linolenico (Omega 3), 36.80\% de ácido graso esencial linoleico (Omega 6) y $8.28 \%$ de ácido oleico (Omega 9). Además tiene el más bajo contenido de ácidos grasos saturados: $6.39 \%$ en promedio, $3.85 \%$ de palmítico y $2.54 \%$ de esteárico.

Actualmente el Sacha Inchi se comercializa, en su mayor porcentaje, en la forma básica; vale decir, granos tostados (similar al maní) que facilmente se encuentran en la mayoría de las tiendas naturistas, en tanto que en autoservicios se comercializa el aceite, que en su fase de industrialización está dirigido a un segmento pequeño y selectivo de la población limeña. La investigación revela tambien que se pueden fabricar y comercializar otros productos hechos sobre la base del Sacha Inchi. Así, las opciones de su comercialización van desde el inchicapi, ají de sacha inchi, cutacho, mantequilla
\end{abstract}

(*) Doctorado en Ciencias Administrativas, Magíster en Gestión Empresarial y Licenciado en Administración. Docente de Pre y Post Grado de la UNMSM. Profesor visitante de las Escuelas de Post Grado de las universidades UNP (Piura), UNT (Trujillo), UNLG (Ica), UNA (Puno) y UNSCH (Ayacucho). Consultor en Gestión Humana y Comportamiento Organizacional.

$(* *)$ Licenciada en Administración. Directora Académica de la Facultad de Ciencias Administrativas UNMSM y Docente Permanente de la UNMSM. 
de sacha inchi, inchi cucho, tamal de sacha inchi, turrón, snacks, entre otros; rubros en los cuales la ciudad de Lima todavía es un mercado cautivo por madurar. En todas ellas, las estrategias de promoción y publicidad deben resaltar las propiedades nutritivas e incluso curativas del Sacha Inchi. Al respecto, la investigación de fuentes secundarias revela que el consumo frecuente de esta oleaginosa reduce la obesidad y favorece los tratamientos contra el cáncer, diabetes, enfermedades del corazón, presión arterial, disfunción eréctil y estrés. Incluso hay una investigación que postula que el Sacha Inchi previene el Alzheimer, mejora la visión, mejora el desarrollo infantil, refuerza la concentración mental y fortalece el sistema inmunológico.

PALABRAS CLAVE: Comercialización, Estrategias, Posicionamiento, Sacha Inchi, Demanda, Oferta, Variedades.

\section{ABSTRACT}

This research has as purpose to develop viable strategies for positioning, marketing the sacha inchi and its derivatives in the market of the big Lima.

The Inchi Sacha is a native plant of the peruvian Amazon, first described as a species in 1753 by naturalist Linneo, hence its scientific name Plukenetia volubilis Linneo.

In Peru is known of its existence from the empire. Thus, until today has taken different names: Sacha Inchi, quechua word meaning peanut mount; Amui a term used by the aboriginal tribes of the Amazon, and the Inca Peanut.

Today, also known as the Inca Peanuts, is the natural oilseed with a higher content of omega, an essential fatty acid for human life. Composed: $48.60 \%$ of essential fatty acid alpha linolenic (Omega 3), $36.80 \%$ of essential fatty acid alpha linoleic (Omega 6 ) and $8.28 \%$ of oleic acid (omega 9). It also has the lowest content of saturated fatty acids: $6.39 \%$ on average, $3.85 \%$ of palmitic and $2.54 \%$ of stearic.

Nowadays the Sacha Inchi is sold at its highest percentage in the basic form, ie, toasted beans (similar to peanuts) which is easily located in most healthy food stores, while supermarkets sell oil, which in stage of industrialization is aimed at a small and selective segment of the population of Lima. The research also shows that we can manufacture and market other products made on the basis of Sacha Inchi. This way, the marketing options are: Inchicapi, chili of sacha inchi, cutacho, sacha inchi butter, inchi cucho, acha inchi tamale, nougat, snack, among others; items in which the city of Lima, is still a captive market to mature. In all, the advertising and promotion strategies should emphasize the nutritional and even healing properties of Sacha Inchi. In this regard, secondary research shows that frequent consumption, reduces obesity and favours the cancer treatment, diabetes, heart disease, blood pressure, erectile dysfunction and stress. Even, there is a research that posits that Sacha Inchi prevent Alzheimer's, improve vision, improve child development, enhances mental focus and strengthens the immune system.

KEY WORDS: ...

\section{INTRODUCCIÓN}

Hoy en día el cuidado de nuestro cuerpo y la naturaleza se han convertido en una de las formas casi obligatorias de existencia. La humanidad se preocupa y busca alternativas de consumo, en lo posible de productos naturales, que garanticen nutrientes y prevengan enfermedades futuras, además que ayuden a la preservación de la ecología. En este contexto, el Sacha Inchi es la oleaginosa nueva y más grande descubierta por la ciencia médica en los últimos años, que contiene propiedades benéficas para la salud, dado que es un producto natural.
El presente trabajo está referido al proceso de producción y comercialización del Sacha Inchi, ya sea en su forma básica (en granos similares al maní), o en representaciones o variedades diversas (aceite, inchicapi, ají de sacha inchi, cutacho, mantequilla de sacha inchi, inchi cucho, tamal de sacha inchi, turrón, snacks, crema antiarrugas, etc., todo fabricado en base a sacha inchi), pero todos ellos con una caracteristica en común, que es su naturalidad al $100 \%$.

Los resultados que se obtuvieron a lo largo de la investigación recomiendan que paralelo a la comercialización, las empresas del sector deben 
trabajar en el posicionamiento del producto, resaltando las propiedades alimenticias y curativas que se encuentran en dicha planta.

En esta parte, toda investigación que pretende encuadrarse en el campo científico, debe observar una metodología de trabajo y definir claramente los objetivos de investigación. Al respecto, los siguientes son los objetivos propuestos en la presente investigación:

- Demostrar que las estrategias de posicionamiento y comercialización del Sacha Inchi que vienen desarrollando las empresas actualmente todavía son aisladas e incipientes.

- Proponer la estrategia o estrategias articuladas y viables para desarrollar el mercado de consumo del Sacha Inchi y sus derivados en la gran Lima.

La presente investigación contiene en su primera parte el constructo teórico existente hasta la fecha, seguida de los hallazgos encontrados en el trabajo de campo, siendo necesario en esta parte plantear las hipótesis, identificar las variables y los indicadores. En la parte final se presentan las conclusiones así como las recomendaciones, esperando que las empresas y las instituciones públicas tomen nota de ello.

\section{INFORMACIÓN PARA EL CONSTRUCTO TEÓRICO}

\section{Características y atributos del Sacha Inchi}

El Sacha Inchi es un producto natural desconocido entre la mayoría de la población peruana. Por ello se presenta con detalle información de esta planta amazónica:

- Nombre científico: Plukenetia Volubilis Linneo.

- Nombre vulgar: Sacha Inchi, Sacha Maní, Inca Inchi, Maní del Inca

- Periodicidad: Producción permanente por 5 años de vida útil, extendible hasta los 10 años.

- Precoz: Planta vigorosa, primera cosecha a los 6 u 8 meses.

- Rústica: No es exigente en suelos, muy poco susceptible al daño de plagas y enfermedades.

- Trepadora: Siendo enredadera y exigente en luz, requiere de tutores y de tendales de alambres.

\section{- Morfología General:}

- Planta: Trepadora, voluble, semi-leñosa, de altura indeterminada.

- Hojas: Son alternas, de color verde oscuro, oval elípticas, aseruladas y pinnitinervias, de 9 a $16 \mathrm{~cm}$ de largo y 6 a $10 \mathrm{~cm}$ ancho. El ápice es puntiagudo y la base es plana o semi - arriñonada.

- Flores: En Sacha Inchi se observan 2 tipos de flores: las masculinas, que son pequeñas, blanquecinas, dispuestas en racimos; y las femeninas, que se encuentran en la base del racimo y ubicadas lateralmente de una a dos flores.

- Fruto: Es una cápsula de 3.5 a $4.5 \mathrm{~cm}$ de diámetro, con 4 lóbulos aristados (tetra lobados) dentro de los cuales se encuentran 4 semillas. Excepcionalmente algunos presentan cápsulas con 5 a 7 lóbulos.

- Semilla: Es ovalada, de color marrón oscuro, ligeramente abultada en el centro y aplastada hacia el borde. El diámetro fluctúa entre 1.3 y $2.1 \mathrm{~cm}$.

\section{Investigaciones y esfuerzos comerciales y de industrialización en el Perú}

La primera mención científica del Sacha Inchi fue hecha en 1980 a consecuencia de los análisis de contenido graso y proteico realizados por la Universidad de Cornell en Estados Unidos, los que demostraron que las semillas del Sacha Inchi tienen alto contenido de proteínas (33\%) y aceite (49\%); pero recién en el año 2000 se le estudia con rigor científico y gracias al apoyo de científicos europeos y a la colaboración de la Universidad Agraria de la Molina (UNALM) se confirmó la presencia de Omega, proteínas y una gran cantidad de antioxidantes en las semillas. La Tabla №1 es ilustrativa al respecto:

Tabla № 1

Contenido de proteínas y ácidos grasos en Sacha Inchi y otras oleaginosas

\begin{tabular}{|l|c|c|c|c|c|c|c|}
\hline \multirow{2}{*}{$\begin{array}{c}\text { Nutriente } \\
(\%)\end{array}$} & \multicolumn{7}{|c|}{ Semillas de oleaginosas } \\
\cline { 2 - 9 } & Sacha & Soya & Maíz & Girasol & Algodón & Palma & Oliva \\
\hline Proteínas & 29 & 28 & & 23 & 24 & & \\
\hline Aceite total & 54 & 19 & & 45 & 48 & & \\
\hline Palmítico & 3.85 & 10.5 & 11 & 12 & 7.5 & 45 & 13 \\
\hline Esteárico & 2.54 & 3.2 & 2 & 2.2 & 5.3 & 4 & 3 \\
\hline oleico & 8.28 & 22.3 & 28 & 43.3 & 29.3 & 40 & 71 \\
\hline linoleico & 36.8 & 54.5 & 58 & 36.8 & 57.9 & 10 & 10 \\
\hline Linolénico & 48.61 & 8.3 & 1 & & & & 1 \\
\hline
\end{tabular}


En el Perú la empresa Agroindustrias Amazónicas ha identificado variedades de Sacha Inchi hasta con $54 \%$ de aceite. Tales variedades presentan un importante contenido de aminoácidos esenciales y no esenciales. Esta oleaginosa de por sí es rica en vitaminas A y E, en cantidades suficientes para la salud humana. Contiene 562 calorías y su índice de yodo es alto: 192 .

Dentro de las diversas propiedades del Sacha Inchi adquieren mayor importancia aquellas referidas a las propiedades curativas. Existen informes donde se afirma que aquellos que la consumen con regularidad reducen la obesidad y favorece los tratamientos contra el cáncer, diabetes, enfermedades del corazón, presión arterial, disfunción eréctil y estrés. Después de todo, la ciencia médica ha confirmado que el Sacha Inchi previene el Alzheimer, mejora la visión, mejora el desarrollo infantil, refuerza la concentración mental y fortalece el sistema inmunológico, dado que el $49 \%$ de su composición química corresponde a la omega 3.

En el 2001, la empresa Agroindustrias Amazónicas dio inicio al Proyecto Omega para cultivar esta planta dentro de un concepto tecnológico, comercial, industrial y social, conjugando la mentalidad de la zona con el ritmo del crecimiento y desarrollo económico del mercado global. En el 2004 logró su primera medalla de oro en el concurso Aceites de Semillas del Mundo realizado en París; y ha sido merecedor de otros dos reconocimientos por la innovación tecnológica en su extracción.

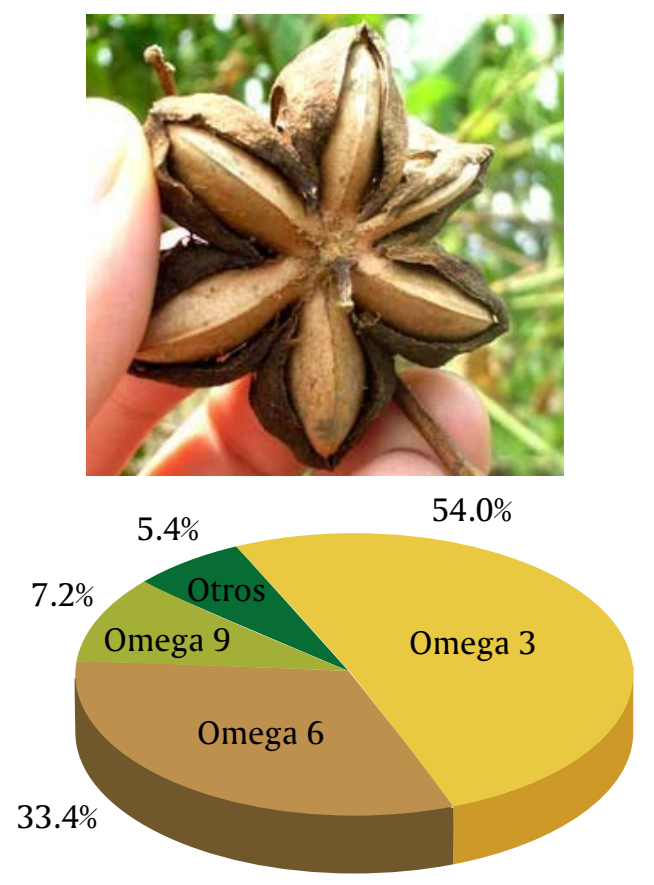

De la misma forma, el Sacha Inchi es el producto vegetal con la menor cantidad de aceites saturados, lo que difícilmente se puede encontrar en otros productos. Ello se debe a sus altos componentes de Omega 3 y 6 que ayudan, según estudios científicos, a prevenir los ataques al corazón. Todo ello configura un escenario favorable para que dentro de poco la población limeña cambie sus preferencias, convirtiendo al Sacha Inchi como una oleaginosa de mayor aceptación.

\section{Propiedades curativas}

Dentro del análisis, el Sacha Inchi posee uno de los más altos contenidos de ácidos grasos polinsaturados entre todas las semillas de consumo humano. Alcanza un promedio de $48 \%$ de ácido linolénico (Omega 3), alrededor del $37 \%$ de ácido linoleico (Omega 6) y aproximadamente un $8 \%$ de ácido oleico (Omega 9).

La propiedad del Omega $3(\Omega 3)$ es evitar que se formen otros compuestos en el organismo humano ya que, al incrementarse la tendencia a la coagulación de la sangre, evita la inflamación, constricción de las arterias y predisposición a arritmias. Por tanto, una dieta con el adecuado balance de $\Omega 3$ es necesaria para una buena salud. Además, disminuye los valores de triglicéridos, que son grasas que están en la sangre, evitando así el endurecimiento de las arterias.

La Omega 3 también se encuentra en los pescados. Entre los peces cuyas carnes tienen mayor cantidad de Omega 3 (entre 24\% y 39\%) tenemos a la anchoveta, sardina, jurel, caballa, merluza; es decir los pescados consumidos por la mayoría de la población de bajos recursos. Esto que puede ser una ventaja, para un buen sector de la población de mayores recursos no siempre es así, ya que a este grupo de personas no les gusta consumir los pescados antes mencionados por su olor penetrante, de tal forma que prefieren consumir los llamados pescados blancos como la corvina, lenguado, mero, chita, tollo, robalo, etc., cuya cantidad de Omega 3 es mucho menor. Además a algunas personas la carne de pescado les provoca reacciones alérgicas. Es por ello que el Sacha Inchi no solo es una alternativa alimenticia frente a los pescados, sino que es la opción más rica en aceite que todas las demás semillas oleaginosas que existen como el girasol, oliva, soya, maíz, palma y maní. 
Investigaciones serias precisan que las propiedades curativas del Sacha Inchi son las siguientes:

- Ayuda a transportar el oxígeno de las células de la sangre a los tejidos.

- Colesterol: Prevención en la saturación de las arterias al ayudar a reducir el riesgo de sufrir de enfermedades coronarias.

- Actúa como antioxidante: Regulación de la presión de los ojos, ligamentos y arterias, así como una mejora de la respuesta inmunológica mediata.

- Artritis: Reducción de la inflamación en las arterias.

- Depresión/ Salud mental: Mantenimiento de la fluidez y rigidez de las membranas celulares.

- Hipertensión: Reducción de los triglicéridos en el flujo sanguíneo.

- Diabetes/ Pérdida de peso: Regulación de los niveles de azúcar en la sangre.

- Regulación de las trasmisiones nerviosas y de comunicación.

\section{HIPÓTESIS Y VARIABLES EN LA PRESENTE INVESTIGACIÓN}

\section{Hipótesis}

La hipótesis de trabajo en la presente investigación fue la siguiente:

"Si se incorporan otras estrategias de comercialización del Sacha Inchi a las actuales, se puede lograr un mayor posicionamiento en los clientes potenciales de la gran Lima”.

\section{Variables}

Las variables identificadas en la presente investigación fueron las siguientes:

- Variable Independiente: Nuevas estrategias de comercialización del Sacha Inchi.

- Variable Dependiente: Mayor posicionamiento en los clientes potenciales de la gran Lima.

\section{MÉTODOS Y RESULTADOS DE LA INVESTIGACIÓN}

\section{Metodología para el trabajo de campo}

Dado que el Sacha Inchi no es un producto muy conocido y de consumo masivo, para la recopila- ción de la información primaria se ha convenido en realizar entrevistas a los representantes de los productores y empresas del sector, ubicados tanto en Lima como en las zonas de Selva y ceja de Selva. Por el lado de la demanda se aplicó una encuesta ad hoc a los potenciales consumidores con residencia en la ciudad de la Lima, en una cantidad de 200 personas, aproximadamente. La identificación y selección de las personas a encuestar fue en forma aleatoria y por conveniencia.

Para el caso de los representantes empresariales se han tenido en cuenta a las siguientes empresas:

- Agroindustrias Amazónicas.

- Reátegui Hidalgo Lincoln.

- Agro Selva.

- Pakari Agro.

- Agro Omega 3.

- Industrias Roda.

- Negocios Agroindustriales Loreto SAC.

Como las entrevistas en la mayoría de los casos fueron no estructuradas, se tomaron en cuenta los puntos siguientes a modo de guía o pauta de la conversación:

A nivel de productores e industriales:

- Conocimiento y estudios del mercado.

- Medios y canales de comercialización.

- Precios actuales y proyectados de productos.

- Producción actual.

- Predisposición y disponibilidad para incorporar valor agregado al producto.

- Capacidad potencial para 1, 2 y 5 años.

- Fortalezas y debilidades internas.

- Oportunidades y amenazas externas.

- Capacidad de planta.

A nivel de consumidores:

- Conocimientos de los atributos o bondades del Sacha Inchi.

- Rango de precios de compra.

- Frecuencia de consumo o compra.

- Lugares o establecimientos de compra. 
- Facilidades o dificultades de compra.

- Productos, variedades y presentaciones que compra.

- Medios o formas sugeridos para adquirir los productos del Sacha Inchi.

Las informaciones recabadas con las entrevistas y las encuestas fueron complementadas con información proveniente de fuentes secundarias (noticias, boletines, reportes, publicaciones de instituciones públicas y privadas, etc.).

\section{Análisis y discusión de resultados}

Para tener una idea cabal acerca del mercado y de la competencia es pertinente analizar el mercado, definir el perfil de los potenciales clientes y, por supuesto, diseñar las estrategias de comercialización

\subsection{Análisis del mercado}

El mercado del Sacha Inchi en la Gran Lima por ahora está conformado por aquellos clientes que consumen el aceite y otros que lo consumen en su forma básica, es decir la tostada del Sacha Inchi, similar al maní, que se vende fundamentalmente en establecimientos naturistas.

Por otro lado, las empresas más representativas que abastecen con el aceite de Sacha Inchi al mercado nacional-fundamentalmente Lima-y al internacional son: Agroindustrias Amazónicas, seguida por Negocios Agroindustriales Loreto SAC, Agro Omega 3 e Industrias Roda, respectivamente. En Lima lo que se observa es que los productos son vendidos a través de casas naturistas (tostadas y otros variantes) y autoservicios (principalmente el aceite, con marcas específicas).

En provincias se presentan grandes opciones de desarrollo en los próximos años por su potencialidad de consumo. Las ventajas comparativas para el crecimiento de la demanda interna están dadas por las propiedades alimenticias y curativas del Sacha Inchi que, a su vez, guardan relación directa con las tendencias en las preferencias de los consumidores. Hay un crecimiento significativo en el mundo y en nuestro país, en preferir en el consumo los productos light o dietéticos.

\subsection{Perfil de potenciales clientes}

Son clientes potenciales todas las personas que se preocupan por su salud así como las empresas que comercializan los productos light o dietéticos.
La distribución de tales productos se hace a través de las casas naturistas o de los autoservicios. Estos puntos de venta están dirigidos a las personas que por el ritmo de trabajo o la vida intensa que llevan están propensos a adquirir dolencias y males, y pueden ser víctimas de ataques al corazón, gastritis, ansiedad, tensión y depresión, entre otras; personas que trabajan en oficinas, no hacen deporte y consumen muchas calorías y grasa saturada, dolencias que no diferencian edades, sexos, ni mucho menos niveles socio-económicos.

Estas personas, al adquirir conciencia de su situación, se preocupan en consumir alimentos dentro de la tendencia bio (productos naturales que carecen de elementos químicos). Esta es una tendencia muy acentuada de estos tiempos, ya que quienes están preocupados por tener una vida saludable siempre están revisando qué alimentos consumir y cuáles evitar, tratan de consumir alimentos ricos en sustancias que eviten ciertos males peligrosos como la insuficiencia respiratorias, las cardiopatías, buscando sobre todo que fortalezcan el sistema inmunológico que es el encargado de combatir a los agentes como hongos, virus y bacterias que causan enfermedades. Este mismo sistema reforzado también evita el estrés y el envejecimiento, por esto los clientes actuales y potenciales son aquellos que tienen la tendencia de prevenir y luchar contra los males indicados, quienes valorizan cada vez más el origen del producto y sus condiciones de producción que son los factores que determinan las cualidades del producto.

\subsection{Posicionamiento del Sacha Inchi}

El siguiente cuadro ilustra el nivel de conocimiento del Sacha Inchi entre las personas entrevistadas:

Cuadro No 1

NIVEL DE CONOCIMIENTO DEL SACHA INCHI

\begin{tabular}{|l|l|}
\hline & $\%$ \\
\hline Sí conocen & 17.00 \\
\hline No conocen & 62.00 \\
\hline No saben/ No opinan & 21.00 \\
\hline TOTAL & 100.00 \\
\hline
\end{tabular}

En las entrevistas a los consumidores potenciales de la gran Lima, comprobamos que todavía el producto no es conocido. A excepción del $17 \%$ que manifestaron conocer, un $62 \%$ no tiene refer- 
tencias del producto. Esta cifra seguramente es mayor si en ella se incorpora también a aquellos que no opinaron.

A nivel de los entrevistados que sí conocen, encontramos que consumieron el Sacha Inchi en cualquiera de las siguientes formas:

Cuadro $\mathrm{N}^{\circ} 2$

MODALIDADES DE CONSUMO DEL SACHA INCHI

\begin{tabular}{|l|r|}
\hline Productos & \multicolumn{1}{c|}{$\%$} \\
\hline Tostado & 32.00 \\
\hline Aceite & 24.00 \\
\hline Mantequilla de Sacha Inchi & 13.00 \\
\hline Turrón de Sacha Inchi & 12.00 \\
\hline Snacks & 8.00 \\
\hline Tamal de Sacha Inchi & 7.00 \\
\hline Ají de Sacha Inchi & 8.00 \\
\hline Otros productos & 17.00 \\
\hline TOTAL & $>=100$ \\
\hline
\end{tabular}

Como vemos, la mayoría de los que conocen han consumido en su forma natural (32\%), y el $24 \%$ como aceite en sus dietas alimenticias. En las otras presentaciones los consumos todavía no son representativos.

Estos datos corroboran nuestra hipótesis en el sentido que el nivel de posicionamiento del Sacha Inchi es todavía muy precario. Por ello creemos que una de las razones de la asociatividad que se debe promover entre los productores e incluso las empresas que industrializan es precisamente el interés de posicionar el producto a través de una promoción sostenida, explicando fundamentalmente las propiedades nutritivas y curativas entre los cliente potenciales.

\subsection{Estrategias de comercialización}

Las estrategias que se vienen utilizando para comercializar el Sacha Inchi en el mercado de la gran Lima se encuadran dentro de los procedimientos clásicos que aconseja la doctrina comercial. Por ello interesa identificar a los variables y elementos que intervienen en el proceso de comercialización:

a) Puntos de venta: El consumidor de Lima solo puede adquirir los productos de Sacha Inchi a través de establecimientos especializados en venta de productos naturistas y autoservicios.
Gráfico № 1

Dónde compraría los productos derivados del Sacha Inchi

En tiendas naturistas

En super mercados

$\square$ Por internet

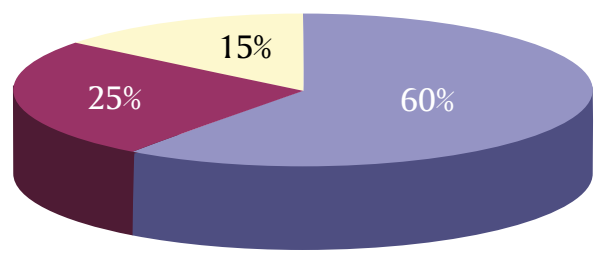

Como podemos observar, las personas relacionan al Sacha Inchi como un producto naturista, por ello un $60 \%$ de los encuestados manifestaron que realizarían sus compras en establecimientos naturistas, en tanto que un $25 \%$ contestaron que compraría en autoservicios. Finalmente, un 15\% lo harían a través del internet.

b) Presentaciones y variedades: A la fecha la forma cómo se consume el Sacha Inchi se reducen a productos tostados (similar al maní), aceite y snacks. Los primeros en casas naturistas y los segundos en autoservicios.

c) Canales de distribución: Como en los tostados no hay un valor agregado, los productores venden directamente a los acopiadores, quienes transportan al mercado limeño para distribuir a las casas naturistas; en tanto en el caso del aceite de Sacha Inchi, que exige para su procesamiento de una tecnología moderna, son las propias empresas industriales las que colocan el producto en los autoservicios con sus propias marcas. Tales empresas son: Agroindustrias Amazónicas, Negocios Agroindustriales Loreto SAC e industrias Roda.

d) Nuevos productos y presentaciones de aceptación futura: Hay una variedad de productos y presentaciones que las empresas tienen en cartera, que pueden tener una gran acogida en el mercado limeño. Por nuestro lado, como parte de la presente investigación, hicimos algunas pruebas piloto de uso o degustación, contando con la aceptación de los encuestados. Los productos posibles de fabricarse teniendo como insumo al Sacha Inchi, son:

- Sacha Inchi en cápsulas.

- Cremas anti-arrugas.

- Mantequilla de Sacha Inchi.

- Turrón de Sacha Inchi.

- Snacks.

- Harina de Sacha Inchi. 
- Torta proteica.

- Tamal de Sacha Inchi.

- Inchicapi.

- Ají de Sacha Inchi.

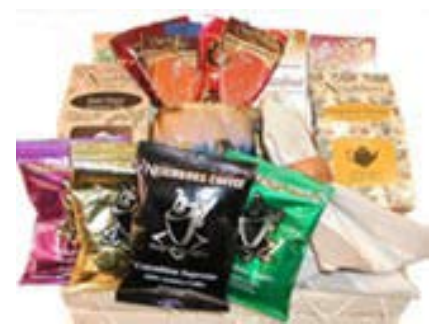

Como se puede apreciar en los 6 primeros se requiere un nivel de industrialización, por tanto serán las empresas las encargadas de su producción y comercialización; en tanto que para los siguientes productos, como no se requiere mayor tecnología, podrían ser los mismos productores quienes den el valor agregado a su producción básica. En esta parte, el Sacha Inchi en cápsulas y los snacks son los que tendrían mayor preferencia con $25 \%$ y $20 \%$, respectivamente.

Gráfico № 2

Nuevas presentaciones de Sacha Inchi

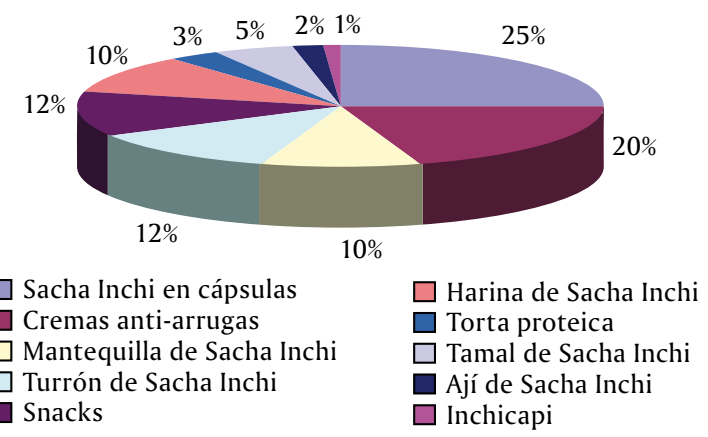

En relación a la frecuencia de consumo, el $30 \%$ de los entrevistados manifestaron consumir siempre (18\%) y casi siempre (12\%), en tanto que el $60 \%$ dicen que todavía no consumen; este segmento es el que representa una potencialidad de consumo a futuro.

Gráfico № 3

Frecuencia de consumo de productos derivados del Sacha Inchi

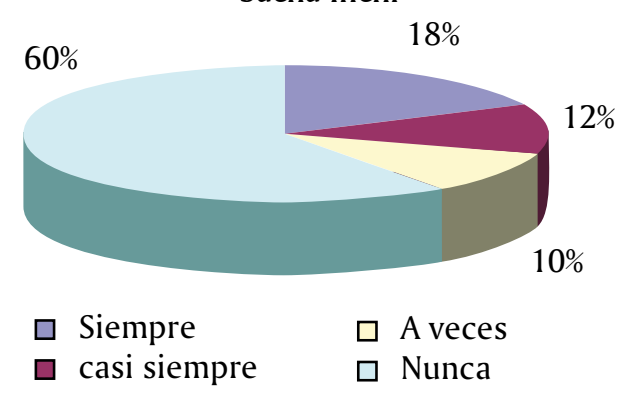

En relación a las características mejor valoradas entre los encuestados encontramos que el $45 \%$ valora al sabor del producto, seguido del $25 \%$ que aprecia el olor. Un $30 \%$ de los encuestados valoran las características de presentación, color y textura. Esta posición es coherente si tenemos en cuenta que el mayor atributo del Sacha Inchi y los productos derivados son sus características nutritivas, curativas y alimenticias.

Gráfico № 4

Características mejor valoradas por los encuestados Textura

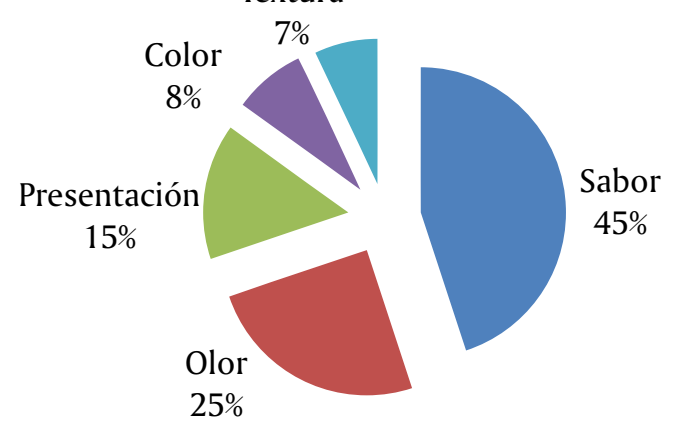

Otro hecho a destacar en esta parte es la prueba piloto que se aplicó en forma aleatoria a algunos encuestados, principalmente para determinar las preferencias en algunas presentaciones para su industrialización futura. Así, el siguiente gráfico muestra que el $40 \%$ de los encuestados manifiestan preferir al producto en su estado original, es decir tostado. Otro tanto manifestó preferir en snack derivado del Sacha Inchi. Finalmente, hay un $20 \%$ que estaría dispuesto a consumirlo como torta proteica. Para la fabricación de la torta proteica se requiere como insumo la harina proteica de Sacha Inchi. Al industrializar el producto previamente para obtener la harina se abriría una línea interesante, dado que se puede utilizar en una amplia variedad de productos y presentaciones.

Gráfico № 5

Prueba de preferencia

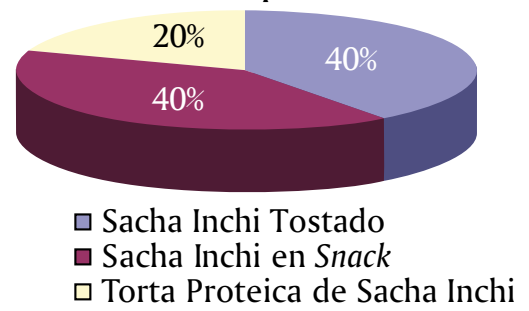

\subsection{Estrategia de precios}

Los precios a los cuales compran los consumidores de Lima no los controla ni el productor 
de la Selva, ni el industrial que vende a los autoservicios. Como vivimos en una sociedad de libre mercado el precio lo define el mercado, por lo que en gran medida el precio de este producto se ve seriamente incrementado por los costos de transporte. Sin embargo, a modo de comparación con otros productos similares, el precio se convierte en una barrera de entrada para los competidores potenciales, tal como podemos apreciar en el siguiente cuadro:

\begin{tabular}{|c|c|c|c|}
\hline \multicolumn{4}{|c|}{ Cuadro № 3} \\
\hline SACHA INCHI & \multicolumn{3}{|c|}{ PRECIOS } \\
\hline $\begin{array}{l}\text { Variedad: } \\
\text { Tostado }\end{array}$ & $\begin{array}{l}\text { Emp. Agro } \\
\text { Selva }\end{array}$ & $\begin{array}{c}\text { Casa } \\
\text { naturista }\end{array}$ & $\begin{array}{c}\text { Por } \\
\text { internet }\end{array}$ \\
\hline $\begin{array}{l}\text { 25gr. } \\
100 \mathrm{gr} .\end{array}$ & $\begin{array}{l}\text { S/. } 1.80 \\
\text { S/. } 7.00\end{array}$ & $\begin{array}{l}\text { S/. } 2.20 \\
\text { S/. } 7.00\end{array}$ & $\begin{array}{l}\text { S/. } 2.70 \\
\text { S/. } 10.00\end{array}$ \\
\hline $\begin{array}{l}\text { MARCAS DE SAC } \\
\text { INCHI }\end{array}$ & \multicolumn{3}{|c|}{ PRECIOS EN AUTOSERVICIOS } \\
\hline Aceite $250 \mathrm{grs}$ & Metro & Plaza Vea & Wong \\
\hline INCA INCHI & S/. 30.00 & $S / 30.00$ & S/. 32.00 \\
\hline
\end{tabular}

\subsection{Presentación de los productos}

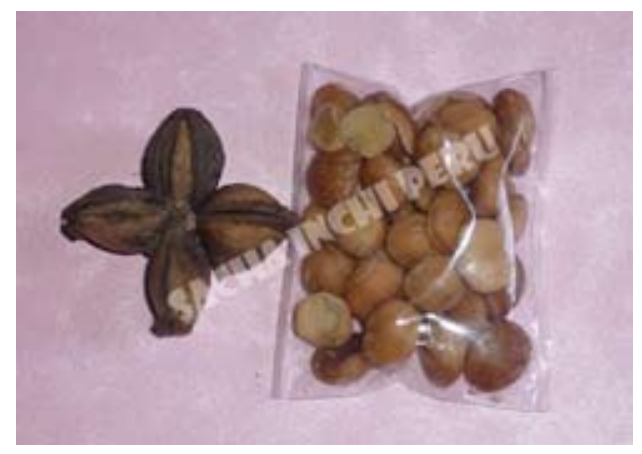

$25 \mathrm{gr}$.

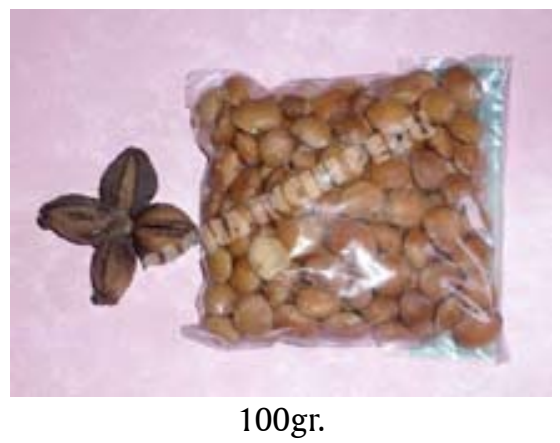

\subsection{Promoción y publicidad}

Las estrategias promocionales deben estar dirigidas a los clientes potenciales, para ello creemos que la publicidad debe recurrir al uso de afiches y gigantografías que bien pudieran colocarse en lugares estratégicos dentro de las tiendas naturistas. Eso mismo se hará en los restaurantes de comida natural.
A nivel de los autoservicios una forma efectiva de publicitar sería la degustación directa a través de impulsadoras ubicadas dentro de las tiendas. Esta estrategia promocional debe ser reforzada con trípticos y otros impresos, donde se expliquen ampliamente las bondades y las propiedades nutritivas y curativas del Sacha Inchi. Otra de las formas de publicitar sería publicar avisos en revistas o publicaciones especializadas ligadas al público objetivo. Un slogan oportuno que puede servir para publicitar el Sacha Inchi podría ser: "Sacha Inchi, salud y más vigor en tus alimentos".

Finalmente, el Internet es otro medio donde se debe publicitar en forma sostenida, creando blogs o listas de interés donde se pueden mencionar las bondades del producto y que han sido detalladas en la presente investigación.

\subsection{El mercado internacional en perspectiva}

Creemos que para la comercialización del Sacha Inchi y sus derivados el mercado cautivo no solo es la gran Lima. Si hacemos un análisis en perspectiva veremos que el mercado internacional se presenta como una opción muy interesante que los comercializadores deben tomar nota en el futuro cercano. Los datos del año 2006 que

\section{Gráfico № 6}

Exportaciones de Sacha Inchi y sus derivados según empresa exportadora 2006

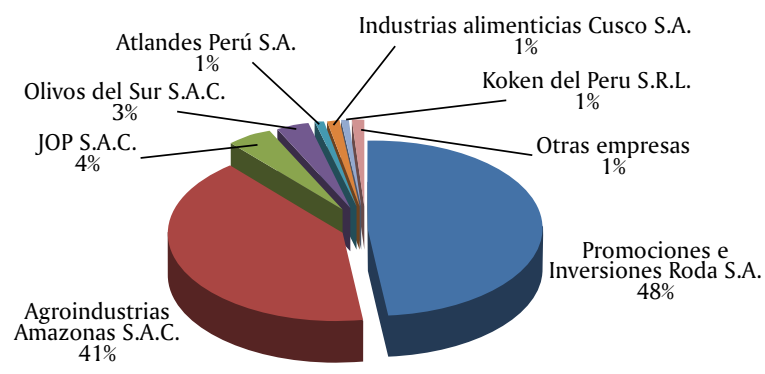

Fuente: Superintendencia Nacional de Administración Tributaria Elaboración: Biocomercio Perú / PROMPEX

\section{Gráfico № 7}

Exportaciones de Sacha Inchi y sus derivados según principales países de destino 2006

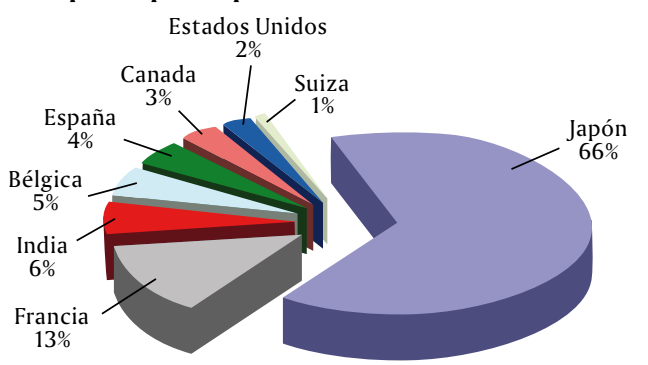

Fuente: Superintendencia Nacional de Administración Tributaria Elaboración: Biocomercio Perú / PROMPEX 
proporciona PROMPEX van en esa línea. Dentro de los países que más consumieron nuestras exportaciones están Japón (64\%), Francia (10\%), y otros países europeos.

\section{CONCLUSIONES Y RECOMENDACIONES}

Luego de investigar el nivel de posicionamiento y comercialización del Sacha Inchi en el mercado de la gran Lima, presentamos algunas ideas fuerza a modo de conclusiones y recomendaciones:

1. La presente investigación sugiere que se pueden establecer alianzas comerciales directas entre productores y consumidores. Actualmente el Sacha Inchi se comercializa en su forma básica, vale decir granos tostados (similar al maní) en tiendas naturistas; en tanto que en autoservicios se comercializa el aceite, que en su fase de industrialización está dirigido a un segmento pequeño y selectivo de la población limeña. La investigación tambien revelan que se pueden fabricar y comercializar otros productos hechos sobre la base del Sacha Inchi. Así, las opciones de su comercialización van desde el inchicapi, ají de Sacha Inchi, cutacho, mantequilla de Sacha Inchi, inchi cucho, tamal de Sacha Inchi, turrón, snacks, entre otros; rubros en los cuales la ciudad de Lima todavía es un mercado cautivo por madurar. En todas ellas, las estrategias de promoción y publicidad deben resaltar las propiedades nutritivas e incluso curativas del Sacha Inchi. Al respecto, la investigación de fuentes secundarias revela que el consumo frecuente reduce la obesidad y favorece los tratamientos contra el cáncer, diabetes, enfermedades del corazón, presión arterial, disfunción eréctil y estrés. Incluso hay una investigación que postula que el Sacha Inchi previene el Alzheimer, mejora la visión, mejora el desarrollo infantil, refuerza la concentración mental y fortalece el sistema inmunológico.

2. El Sacha Inchi es una oleaginosa que tiene propiedades alimenticias y curativas para el ser humano. En su composición se concentra una gran cantidad de Omegas 3, 6 y 9; en 49\%, 37\% y $8 \%$, respectivamente, que hacen de este un producto sui generis que los humanos necesitamos para combatir los males de estos tiempos. Tales propiedades deben ser aprovechadas en estrategias promocionales y publicitarias que demuestren las bondades de esta planta amazónica, que es valorada desde nuestros antepasados pre e incas.

3. Esta investigación también muestra que hay una potencialidad de productos y presentaciones nuevas por aprovechar teniendo como materia prima el Sacha Inchi. Algunos exigen un nivel de industrialización -cremas antiarrugas, snacks, turrones, mantequillas- y otros como el tamal, inchicapi, aji de Sacha Inchi, cutacho e inchi cucho, requieren un valor agregado incipiente para su comercialización. Todo ello configura una potencialidad por explotar a partir del Sacha Inchi, que irá haciéndose realidad en la medida que se posicione el producto natural.

4. En relación a las estrategias de comercialización para el mercado local se sugiere incorporar a las actuales los canales de distribución más directos entre la oferta y la demanda. Como hemos comprobado, las únicas vías por las que acceden los consumidores es a través de acopiadores que distribuyen a las casas naturistas, y a nivel de las industrias en venta directa a los autoservicios. En este punto, urge realizar iniciativas que pasan por un proceso de asociatividad de los productores, quienes aparte de constituir sus propios canales -para evitar encarecer el producto hacia los consumidores finalesbien pueden aprovechar las bondades de la tecnología para realizar ventas directas. En ese sentido, las ventas por internet (el llamado e-comerce) se presentan como una alternativa interesante.

\section{BIBLIOGRAFIA}

Brack, A. (1999). Plukenetia volúbilis L. Diccionario Enciclopédico de Plantas Útiles del Perú. Cuzco, PNUD, 550 pág.

Hazen y Stoewesand. (1980). Resultados de análisis del aceite y proteína del cultivo de sacha inchi. Estados Unidos, Universidad de Cornell.

Manco, Emma. (2006). Cultivo de Sacha Inchi. San Martín, Ministerio de Agricultura, Instituto Nacional de Investigación y Extensión Agraria (INIA). Junio 2006.

Ministerio de Agricultura. (2002). Estadística Agraria Trimestral. Sistema de Información Agraria (SIAG). Lima, MINAG, julio-setiembre 2002. 
Pascual, Ch. G. y Mejía, L. M. (2000). “Extracción y Caracterización de Aceite de Sacha Inchi”. En: Anales Científicos UNALM. Vol. 42, enero - marzo. Lima, UNALM, pág. 144-158.

Proyecto Omega. 2002. El Inca Inchi. Lima, Agroindustrias Amazónicas, 6 pág.

Soukup, J. (1987). Vocabulario de los Nombres Vulgares de la Flora Peruana y Catálogo de los Géneros. Lima, Editorial Salesiana, 436 pág.

Valles, C. (1995). Sacha Inchi, Importante Oleaginosa Selvática. Ciudad, Pura Selva, pág. 40-41.

\section{PÁGINAS WEB}

Aceite de Sacha Inchi: Omega 3 en la Amazonía Peruana. en: $h t t p: / / w w w$. agenciaperu.com

INIEA - SUDIRGEB - EEA. "El Porvenir, Cultivo de Sacha Inchi, Junio 2006 en: http://www. incainchi.es/pdf/1358.pdf

http://www.minag.gob.pe/agricola/pro_ama_sacha. shtml (ficha técnica del Sacha Inchi).

http://www.peru.com/vidasana/idocs/2007/5/28/ DetalleDocumento_411196.asp - 32k. www.agrosanmartin.gob.pe/documentos/dia_costo_ cultivos/items/sacha_inchi.pdf

http://www.proycontra.com. pe/2007/12/30/\%E2\% $80 \% 9$ Csachainchi $\%$ E2\%80\%9D-fue-el-cultivofavorito-del-2007\%E2\%80\%9D/

http://mercadosexportacion.blogspot.com/2008/05/ exportaciones-de-sacha-inchi-crecieron.html

http://www.alimentacion-sana.com.ar/Portal $\% 20$ nuevo/compresano/plantillas/ sachainchi.htm

http://www.agromaz.com.pe/documentos/extraccion caracterizacion_aceite_sacha_inchi.pdf

http://educadis.huascaran.edu.pe/mod/resource/view. php?id $=926$

http://www.americatv.com.pe/cuartopoder/reportajes. asp?id_reportaje $=305 \delta p=1-27 \mathrm{k}$

http://www.inia.gob.pe/SIT/consPR/adjuntos/1418. $p d f$

http://www.elcomercioperu.com.pe/EdicionImpresa/ Html/2007-05-27/ImEcMiNegocio0729123. html - $28 k$ 


\section{MODELO DE ENCUESTA A LOS POTENCIALES CONSUMIDORES}

Sr/Sra/Srta: Esta encuesta es de carácter anónimo y se hace para conocer sus gustos o preferencias en relación a los productos derivados del SACHA INCHI. (Mucho agradeceremos se sirva contestar con la mayor objetividad posible). Gracias por su colaboración.

1. Ha escuchado hablar del Sacha Inchi
a) $\mathrm{Si}$
b) No
c) No sabe/ No opina

2. ¿Con qué frecuencia consume productos derivados del Sacha Inchi?
a) Siempre
c) A veces
b) Casi siempre
d) Nunca

3. ¿Cuál de estas presentaciones de "Sacha Inchi" ha consumido? (Mostrar la tarjeta)
a) Tostado
c) Snacks
e) Otros (Precisar:
b) Aceite
d) Turrones

4. ¿Dónde compraría los productos derivados del Sacha Inchi?
a) Tiendas naturistas
c) Internet
b) Supermercados
d) Otros:

5. ¿Cuál de los productos derivados del Sacha Inchi compraría? (puede marcar más de uno)
a) Sacha Inchi en cápsulas
f) Ají de Sacha Inchi
b) Cremas antiarrugas
g) Torta Proteica
c) Mantequilla Sacha Inchi
h) Tamal de Sacha Inchi
d) Turrón de Sacha Inchi
i) Ají de Sacha Inchi
e) Harina de Sacha Inchi
j) Inchicapi

6. ¿Qué características de las siguientes le llaman la atención en los derivados del Sacha Inchi?
a) Sabor
c) Presentación de Empaque
e) Textura
b) Olor
d) Color 


\section{PRUEBA DE PREFERENCIA}

Esta prueba piloto se realizó solo con personas que aceptaron colaborar en la degustación.

\section{Instrucciones:}

Por favor, pruebe el tostado de SACHA INCHI. A continuación pruebe el Snack de SACHA INCHI. Ahora, pruebe la Torta Proteica de SACHA INCHI.

7. Mencione en orden de importancia los productos degustados. Sugiera las formas de presentación, las características diferenciales y otras consideraciones para la promoción y publicidad de tales muestras:

\begin{tabular}{|l|l|l|}
\hline \multicolumn{1}{|c|}{ PRODUCTO } & ORDEN & COMENTARIO \\
\hline Tostado & & \\
\hline Snack & & \\
\hline Torta proteica & & \\
\hline
\end{tabular}

\section{DATOS DE CONTROL}

Sexo: H( ) M( ) Edad: ............. Ocupación:

Distrito que reside: 